\title{
Synergy Effect of Regional Industrial Agglomeration and Urbanization
}

\author{
Congsheng Wang, ${ }^{1,2}$ Guangxiong Mao, ${ }^{3}$ and Wei-Ling $\mathrm{Hsu}^{3 *}$ \\ ${ }^{1}$ School of Economics and Management, Huaiyin Normal University, \\ No. 111, Changjiang West Rd, Huaian City, Jiangsu 223300, China \\ ${ }^{2}$ School of Economics and Management, China University of Mining and Technology, \\ No. 1, Daxue Rd, Xuzhou City, Jiangsu 221116, China \\ ${ }^{3}$ School of Urban and Environmental Science, Huaiyin Normal University, \\ No. 111, Changjiang West Rd, Huaian City, Jiangsu 223300, China
}

(Received July 20, 2020; accepted January 6, 2021)

Keywords: industrial agglomeration, urbanization, synergy, compound system, synergy effect model

Building on the basic views of synergy theory, we analyze the synergy mechanism between industrial agglomeration (the development of new clusters of industry) and urbanization (the increase in the population and level of development of urban areas). The data sources of some indicators use automatic sensors to collect information on electricity and sewage consumption. With the aid of nonparametric statistical analysis [bootstrap data envelopment analysis (DEA)] and the fuzzy mathematics membership degree concept, we construct a synergy effect measurement model of a composite system, which takes industrial agglomeration and urbanization as subsystems. We evaluate the synergetic development of an industrial agglomeration and urbanization composite system. The results show that the synergetic development level of the Jiangsu industrial agglomeration and urbanization composite system shows regional differences, which are affected by the socioeconomic factors of the two subsystems. They also show that the level of synergetic development of the industrial agglomeration and urbanization complex system is not high and needs to be further improved because it has not reached a stable state. Accordingly, we give some suggestions for enhancing the synergetic development level of industrial agglomeration and urbanization and promoting socioeconomic coordination and sustainable development. These include constructing a planning system for the interactive development of industrial agglomeration and urbanization, enhancing overall interactive development forces of industrial agglomeration and urbanization, guiding industrial agglomeration by urbanization points, applying an agglomeration strategy to optimize industrial layout, and reforming and innovating administrative management systems.

\section{Introduction}

With the improved performance of new sensors, city managers can collect big data such as water consumption, electricity consumption, and sewage treatment for urban governance. Industrialization and urbanization have driven China's current economic development. The *Corresponding author: e-mail: quartback@hotmail.com https://doi.org/10.18494/SAM.2021.3039 
level of China's urbanization has significantly increased. By the end of 2019, the urbanization rate of China's permanent population reached $60.6 \%$, compared with only $41.68 \%$ in 1978 . The development of urban areas is sometimes slower than their urbanization, which results in new cities lacking industrial infrastructure in some places. The 19th National Congress of the Communist Party of China proposed to speed up the implementation of a "new urbanization strategy with the promotion of urbanization as the core and the improvement of quality as the guidance". It is of great significance to explore the relationship between regional industrial agglomeration and urbanization in order to promote the process of urbanization with Chinese characteristics and realize the integrated development of cities. The core of the new development strategy is to promote urbanization to improve the quality of life in cities.

In China, smart telecom sensing is used in major cities. Power supply companies conduct 24hour real-time monitoring of power consumption through an online operation and maintenance platform. This can eliminate the blind areas in information collection through traditional periodic manual inspections and paper-based records, and not only reduce labor costs, but also quickly detect problems and improve overall efficiency. After years of practice, government monitoring agencies have formed three levels of technical products with water quality sensors, communication transmissions, and intelligent control cloud computing platforms as the core, which have been integrated into an overall water quality cloud computing system integration platform. In this study, we applied multivariate analysis (MVA) to establish an index system for the comprehensive evaluation of urbanization. The index system includes population urbanization, economic urbanization, social urbanization, spatial urbanization, and ecological resources as urbanization dimensions and 19 second-level indicators.

\section{Method}

"Synergy" is the relationship between things with harmony and positive matching. "Synergetic development" is a dynamic relationship between things in the state of development that promote each other and move forward synchronously. ${ }^{(1)}$ The main aim of this paper is to evaluate and analyze the collaborative development of industrial agglomeration and urbanization, which play roles as subsystems to form a composite system. The two subsystems take part in the composite system as "order parameters" or "control parameters" in the "self-organization" and promote the transition from disorder to order or from lower order to higher order through synergy to increase the benefits and functionality of the entire system. We used a non-parametric statistical analysis method [bootstrap data envelopment analysis (DEA)] and the concept of fuzzy mathematics to build a synergy effect model of the synergy effect of the composite system of industrial agglomeration and urbanization.

Bootstrap DEA is a non-parametric statistical analysis method used to measure the relative efficiency of a system that is based on DEA. ${ }^{(2)}$ This method uses repeated sampling to simulate the distribution of standard DEA efficiency, which is obtained by traditional DEA. On this basis, the estimated deviation of the standard DEA efficiency is further corrected to obtain the bootstrap DEA efficiency through appropriate iterations. This method also gives the confidence interval of the estimated economic decision unit efficiency. 


\section{Literature Review}

Scholars at home and abroad have carried out deep and extensive research on the relationship between industrial agglomeration and urbanization. Most foreign scholars used theoretical economic models to discuss the relationship and mechanism between industrial agglomeration and urbanization. ${ }^{(3,4)}$ The interaction between industrial clusters and urbanization has been examined from different angles, and a great deal of progress has been made. ${ }^{(3,5)}$ MVA can be complicated by the desire to include physics-based analysis to calculate the effects of variables in a hierarchical "system-of-systems" ${ }^{(6)}$ Often, the desire to use MVA is stalled by the dimensionality of the problem. Most western scholars use theoretical economic models to explore the interrelationship and mechanism between industrial agglomeration and urbanization. ${ }^{(7-9)}$ Oriental scholars have studied the relationship and interactive development of industrial clusters and urbanization from different angles. ${ }^{(3,10)}$ Synergy theory in physics is a comprehensive discipline that has been developed and widely used in the past decades, ${ }^{(11)}$ which mainly studies the mechanism and laws of open systems and forms an ordered structure through the synergy between internal subsystems. The theory focuses on the similarity of various systems from disorder to order. In recent years, some scholars have applied synergy theory to research on industrial agglomeration and urbanization. ${ }^{(10,12)}$ A model of a synergy mechanism for a high-tech industrial cluster framework has been designed, and a path to achieve synergy by constructing an effective system synergizing resources, institutions, agglomeration, competition, and collaboration has been proposed. ${ }^{(13)}$ There has also been research focusing on information resource capacity strategy (IRCS). A system for evaluating the cluster synergy effect of small and medium-sized enterprises and a synergy effect model have also been proposed to conduct empirical analysis and research. Urbanization is divided into five systems: population, economy, society, space, and ecology. The purpose of the systems is to build an index system to evaluate coordinated development and a coupled coordination model to quantitatively measure the level of internal coordinated development of urbanization. ${ }^{(13,14)}$

Previous studies have mainly discussed the interaction between industrial agglomeration and urbanization from qualitative and quantitative perspectives, while the research on the coordinated development of industrial agglomeration and urbanization has only attracted the attention of scholars in recent years, and there is little research on the synergy between the two. In view of this, the aim of this paper is to bring industrial agglomeration and urbanization into a unified analytical framework, and to evaluate the synergetic development level of the two in the province of Jiangsu. This is achieved by establishing a composite system synergetic effect measurement model that can be used as a reference to promote regional industrial agglomeration and urbanization, and thus achieve coordinated socioeconomic development.

\subsection{Synergy effect model}

The synergy effect model of the composite system synergy effect uses two subsystems with long-term interaction as the mutual input and output. Bootstrap DEA is used to estimate and evaluate the development efficiency of each subsystem from a macro perspective. The 
concept of fuzzy mathematics is used to construct a synergy function to measure the degree of synergetic development between the subsystems. If a composite system $S$ includes two subsystems $S_{1}$ and $S_{2}$ that are the mutual input and output, $S=f\left(S_{1}, S_{2}\right)$, where $f\left(S_{1}, S_{2}\right)$ is a composite function. First, using the indicators of $S_{1}$ as the input and the indicators of $S_{2}$ as the output, the standard DEA efficiency value $\tilde{\theta}_{1}$ is estimated by traditional DEA. After multiple iterations using repeated sampling, the estimated bootstrap DEA efficiency is $\tilde{\theta}_{1}$. In the same way, we estimate the bootstrap DEA efficiency with the indicators of $S_{2}$ as the input and the indicators of $S_{1}$ as the output, $\tilde{\theta}_{2}$. Second, by using the concept of fuzzy mathematics, the function $u(\tilde{\theta})=\tilde{\theta}$ is defined, where $\theta$ is the bootstrap DEA efficiency when $S_{1}$ and $S_{2}$ are the input and output systems, respectively. Then, we have $u_{1}=\tilde{\theta}_{1}$ and $u_{2}=\tilde{\theta}_{2} \cdot u_{1}$ and $u_{2}$ are the state synergy degrees of $S_{1}$ to $S_{2}$ and $S_{2}$ to $S_{1}$, respectively, indicating the mutual requirements for the development of the two subsystems. On this basis, the state synergy degree of the two subsystems at time $t$ is defined as follows as the degree of mutual adaptation and collaborative development between the two subsystems:

$$
u_{1,2}(\tilde{\theta}, t)=u_{2,1}(\tilde{\theta}, t)=\frac{\min \left\{u_{1}, u_{2}\right\}}{\max \left\{u_{1}, u_{2}\right\}}=\frac{\min \left\{\tilde{\theta}_{1}, \tilde{\theta}_{2}\right\}}{\max \left\{\tilde{\theta}_{1}, \tilde{\theta}_{2}\right\}}
$$

Third, the industrial agglomeration subsystem and the urbanization subsystem are constantly developing with time. The synergetic development between the two subsystems is a dynamic process that continually evolves with time. Therefore, the dynamic synergy degree of the composite system in the research time interval $\left[t_{0}, t_{n}\right]$ is defined as

$$
u\left(t_{0}, t_{n}\right)=u\left(\tilde{\theta}_{0}, \tilde{\theta}_{1}, \ldots, \tilde{\theta}_{n} ; t_{1}, t_{2}, \ldots, t_{n}\right)=\frac{1}{t_{n}-t_{0}+1} \sum_{i=0}^{n} u\left(\tilde{\theta}_{i}, t_{i}\right)
$$

Here, $u\left(\tilde{\theta}_{i}, t_{i}\right)$ represents the state coordination degree of each subsystem of the composite system at time $t_{i}(i=0,1,2, \ldots, n)$. When $\left[t_{x}, t_{y}\right] \subseteq\left[t_{0}, t_{n}\right]$ and $x \leq y$, if $u\left(t_{0}, t_{x}\right) \leq u\left(t_{0}, t_{y}\right)$, then the degree of synergetic development of the subsystems in the composite system is monotonically increasing. Otherwise, the degree of synergic development is monotonically decreasing, and measures need to be taken to control it. Finally, a judging criterion ${ }^{(15)}$ (as shown in Table 1) is set up to evaluate and analyze the measured synergy degree of the composite system to indicate the synergy status of the composite system.

Table 1

Evaluation table of state synergy of composite system.

\begin{tabular}{lc}
\hline Interval of synergy degree of composite system & State synergy of composite system \\
\hline$u_{1,2}=0$ & Complete non-synergy \\
$u_{1,2}=[0,0.3]$ & Non-synergy \\
$u_{1,2}=[0.3,0.5]$ & Slight non-synergy \\
$u_{1,2}=[0.5,0.8]$ & Slight synergy \\
$u_{1,2}=[0.8,1]$ & Synergy \\
$u_{1,2}=1$ & Complete synergy \\
\hline
\end{tabular}




\subsection{Data sources}

Some data collection is connected to the Internet of Things and automatic monitoring systems. Sensors for monitoring electricity consumption and sewage management have been applied by government monitoring agencies. In this study, we took 13 prefecture-level cities in Jiangsu, China, as cross-sectional samples and analyzed the degree of synergetic development and the level of the industrial agglomeration and urbanization composite system in each city from 2014 to 2018. The data source is the statistical bulletin "Statistical Yearbook of Jiangsu, China".

\section{Analysis Results}

\subsection{Analysis of single system development level}

(1) Measurement of the level of industrial agglomeration in Jiangsu

To measure the degree and level of industrial agglomeration and to implement a crossregional comparison of industrial concentration at the same time, we select the number of nonagricultural employees as indicators, and use the location quotient (LQ) index. ${ }^{(16)}$ LQ is

$$
L Q_{j}=\frac{x_{i j} / \sum_{i=1}^{n} x_{i j}}{X_{i} / \sum_{i=1}^{n} X_{i}}
$$

where $x_{i j}$ represents the number of employees in the $i$ th $(i=0,1,2, \ldots, n)$ industry of the $j$ th region. $X_{i}$ is the $i$ th $(i=0,1,2, \ldots, n)$ industry in the industrial categories. The larger the value of $L Q_{j}$, the higher the level of industrial agglomeration in the $j$ th region. In this paper, LQ is used as an indicator to measure the industrial agglomeration level of each city in Jiangsu. The calculated values of LQ are shown in Table 2.

Table 2

LQ index of each city in Jiangsu from 2014 to 2018.

\begin{tabular}{lccccc}
\hline \multirow{2}{*}{ Region } & \multicolumn{5}{c}{ Year } \\
\cline { 2 - 6 } & 2014 & 2015 & 2016 & 2017 & 2018 \\
\hline Nanjing & 1.056 & 1.052 & 1.040 & 1.034 & 1.030 \\
Suzhou & 1.045 & 1.047 & 1.043 & 1.043 & 1.045 \\
Wuxi & 1.047 & 1.048 & 1.041 & 1.041 & 1.044 \\
Changzhou & 1.031 & 1.032 & 1.027 & 1.029 & 1.031 \\
Zhenjiang & 1.015 & 1.016 & 1.012 & 1.019 & 1.022 \\
Nantong & 0.984 & 0.988 & 0.986 & 0.995 & 1.001 \\
Taizhou & 0.984 & 0.987 & 0.986 & 0.993 & 1.000 \\
Yangzhou & 0.986 & 0.988 & 0.986 & 0.994 & 0.999 \\
Xuzhou & 0.965 & 0.965 & 0.957 & 0.958 & 0.960 \\
Huaian & 0.927 & 0.930 & 0.927 & 0.935 & 0.941 \\
Yancheng & 0.905 & 0.909 & 0.9103 & 0.924 & 0.932 \\
Suqian & 0.897 & 0.906 & 0.9130 & 0.924 & 0.935 \\
Lianyungang & 0.913 & 0.913 & 0.939 & 0.918 & 0.923 \\
\hline
\end{tabular}


From a statistical point of view, the industries in every region of Jiangsu are highly concentrated with regional differences consistent with the level of regional social and economic development. LQ of southern Jiangsu (Nanjing, Suzhou, Wuxi, Changzhou, Zhenjiang) is larger than 1, indicating that the industry of southern Jiangsu is competitive and advantageous. The degree of synergetic development among industry in central Jiangsu (Nantong, Taizhou, Yangzhou) is balanced, and the advantageousness is not as obvious as that in southern Jiangsu. The industry in northern Jiangsu (Xuzhou, Huaian, Yancheng, Suqian, Lianyungang) is relatively less competitive. From a dynamic point of view, during 2014-2018, the overall LQ basically increased, indicating that the industrial agglomeration showed a maturing trend. The change in LQ of the five downtown areas in northern Jiangsu is less than the average level in Jiangsu, indicating that the industrial agglomeration development in northern Jiangsu is relatively slow.

(2) Analysis of comprehensive level of urbanization in Jiangsu

Research on the evaluation of urbanization level began in the 1990s. To overcome the shortcomings of using a single indicator (such as population urbanization rate) representing the level of urbanization, researchers have constructed an index system for comprehensively evaluating urbanization from different perspectives. ${ }^{(4,5,12,17,18)}$ In accordance with the concept of efficient, inclusive, and sustainable development proposed in the report of the Joint Research Group of the State Council Development Research Center and the World Bank in 2014, the system takes into account the new era of urbanization. The composite index method adopts five dimensions of population urbanization, economic urbanization, social urbanization, spatial urbanization, and ecological resource urbanization. The dimensions include 19 indicators to build a comprehensive index system for evaluating urbanization, as shown in Table 3.

(3) Calculation of per capita indicators based on size of permanent population

We mainly use the improved entropy weight method, which is an objective method to determine weights according to the degree of index dispersion. ${ }^{(19)}$ The larger the degree of index dispersion, the smaller the entropy value and the larger the weight. Conversely, the smaller the degree of index dispersion, the larger the entropy value and the smaller the weight. The weight calculation steps are as follows.

(a) Normalize the indicators.

$$
\begin{gathered}
Y_{a b}^{*}=\left(Y_{a b}-\min \left(Y_{a b}\right)\right) /\left(\max \left(Y_{a b}\right)-\min \left(Y_{a b}\right)\right), 1 \leq a \leq m, 1 \leq b \leq n, \\
Y_{a b}^{*}=\left(\max \left(Y_{a b}\right)-Y_{a b}\right) /\left(\max \left(Y_{a b}\right)-\min \left(Y_{a b}\right)\right),
\end{gathered}
$$

where $Y_{i j}{ }^{*}$ is the infinite index of the $b$ th index in a year. Equation (5) is used to calculate the positive index and Eq. (6) is used to calculate the reverse index. After standardization, the range of index values is between 0 and 1 .

(b) Calculate the weight of the indicator in year $a$ under indicator $b$.

$$
P_{a b}=Y_{a b}^{*} / \sum_{a=1}^{m} Y_{a b}^{*}
$$


Table 3

Index system for comprehensive evaluation of urbanization.

\begin{tabular}{|c|c|c|c|c|}
\hline Name & $\begin{array}{l}\text { First-level } \\
\text { indicator }\end{array}$ & Second-level indicator & Unit & $\begin{array}{l}\text { Indicator } \\
\text { attributes }\end{array}$ \\
\hline \multirow{19}{*}{$\begin{array}{l}\text { Index system for } \\
\text { comprehensive evaluation } \\
\text { of urbanization }\end{array}$} & \multirow{4}{*}{$\begin{array}{l}\text { Population } \\
\text { urbanization }\end{array}$} & Urban population proportion & $\%$ & + \\
\hline & & Urban population density & person per $\mathrm{km}^{2}$ & + \\
\hline & & $\begin{array}{l}\text { Proportion of secondary } \\
\text { and tertiary industry employees }\end{array}$ & $\%$ & + \\
\hline & & Urban unemployment rate & $\%$ & - \\
\hline & \multirow{3}{*}{$\begin{array}{l}\text { Economic } \\
\text { urbanization }\end{array}$} & GDP per capita & $\begin{array}{l}10000 \text { yuan per } \\
\text { person }\end{array}$ & + \\
\hline & & $\begin{array}{l}\text { Proportion of secondary } \\
\text { and tertiary industries in GDP }\end{array}$ & $\%$ & + \\
\hline & & Investment in fixed assets per capita & $\begin{array}{l}\$ 10000 \text { yuan per } \\
\text { person }\end{array}$ & + \\
\hline & \multirow{4}{*}{$\begin{array}{c}\text { Social } \\
\text { urbanization }\end{array}$} & $\begin{array}{l}\text { Number of beds in health institutions } \\
\text { per } 10000 \text { people }\end{array}$ & per & + \\
\hline & & Number of students in colleges & 10000 people & + \\
\hline & & Per capita income of urban residents & $\begin{array}{l}10000 \text { yuan } \\
\text { per year }\end{array}$ & + \\
\hline & & Per capita consumption of urban residents & $\begin{array}{l}10000 \text { yuan } \\
\text { per year }\end{array}$ & + \\
\hline & \multirow{3}{*}{$\begin{array}{c}\text { Spatial } \\
\text { urbanization }\end{array}$} & Built-up area & $\mathrm{m}^{2}$ & + \\
\hline & & Land area per capita & $\begin{array}{l}\mathrm{km}^{2} \text { per } 10000 \\
\text { people }\end{array}$ & + \\
\hline & & Urban road area per capita & $\mathrm{m}^{2}$ per person & + \\
\hline & \multirow{5}{*}{$\begin{array}{l}\text { Ecological } \\
\text { resource } \\
\text { urbanization }\end{array}$} & Annual electricity consumption & billion $\mathrm{kWh}$ & - \\
\hline & & $\begin{array}{l}\text { Comprehensive utilization rate of industrial } \\
\text { solid waste }\end{array}$ & $\%$ & + \\
\hline & & Sewage management rate & $\%$ & + \\
\hline & & Urban green coverage & $\%$ & + \\
\hline & & Park area per capita & $\mathrm{m}^{2}$ per person & + \\
\hline
\end{tabular}

(c) Calculate the entropy of the $b$ th indicator.

$$
e_{b}=-(\ln m)^{-1} \sum_{a=1}^{m}\left(P_{a b} \times \ln P_{a b}\right), 0 \leq e_{b} \leq 1
$$

(d) Calculate the difference coefficient of the $b$ th indicator, $g_{b}=1-e_{b}$; the larger the $g_{b}$, the larger the data difference and the larger the weight.

(e) Obtain the index weight.

$$
w_{b}=g_{b} / \sum_{b=1}^{n} g_{b}
$$

Through the above steps, we calculated the weight of each index in 2014-2018 and then used the equation $Z=\sum_{b=1}^{n} w_{b} \times Y_{a b}^{*}$ to calculate the comprehensive urbanization index of each city in Jiangsu. The results are shown in Fig. 1. 


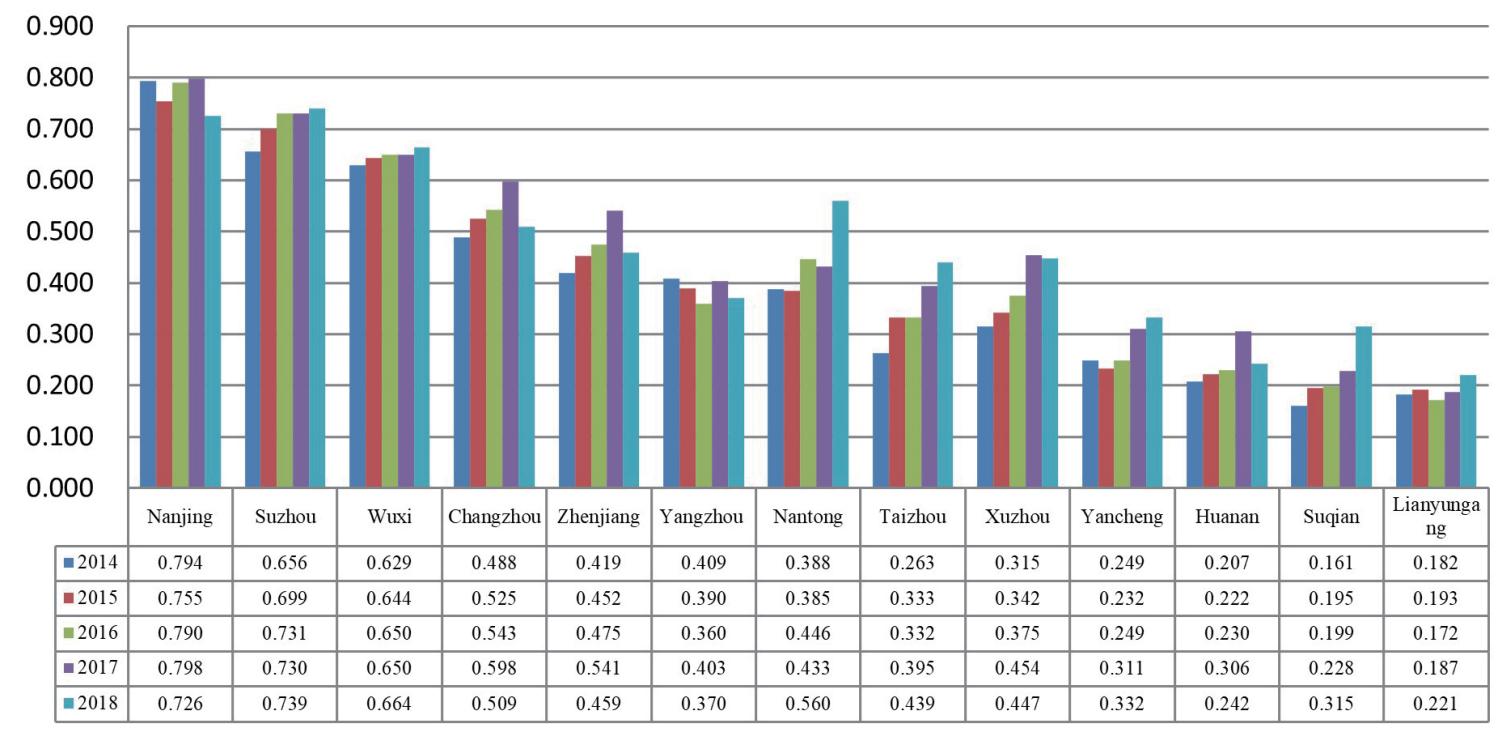

Fig. 1. (Color online) Comprehensive level of urbanization in Jiangsu.

Figure 1 shows that the urbanization development level of the 13 cities in Jiangsu from 2014 to 2018 fluctuated but tended to increase and was related with the level of economic development. Cities with a high level of economic development had a high level of urbanization. The regional differences are obvious. The urbanization level in southern Jiangsu is significantly higher than that in central Jiangsu and northern Jiangsu. The level decreases from south to north. According to the data in 2018, Suzhou has the highest urbanization level while Lianyungang has the lowest. Nantong, which is located in the middle of Jiangsu, has the highest urbanization level, Wuxi is ranked third.

\subsection{Measurement and analysis of the synergy level of the industrial agglomeration and urbanization composite system}

As part of the bootstrap DEA method to measure the mutual development efficiency of various categories of the composite system, Eqs. (1) and (2) were used to calculate the degrees of state synergy and dynamic synergy of the industrial agglomeration and urbanization composite system in each city of Jiangsu. The results in Tables 4 and 5 show that the degrees of state synergy and dynamic synergy have changed significantly, and the change of the state synergy is larger than that of the dynamic synergy.

According to Table 4, the average state synergy of Nanjing and Suzhou was greater than 0.8 between 2014 and 2018. Based on the evaluation criteria for the synergy of the composite system, the composite system of the two cities has a high level of synergetic development. The average state synergy degree of Suqian and Lianyungang is below 0.3, indicating that the two cities are in a non-synergetic state. The average state synergy degree of Yangzhou, Huai'an, 
Table 4

State synergy degree of composite system in cities of Jiangsu obtained using bootstrap DEA.

\begin{tabular}{lccccc}
\hline \multirow{2}{*}{ Region } & \multicolumn{5}{c}{ Year } \\
\cline { 2 - 6 } & 2014 & 2015 & 2016 & 2017 & 2018 \\
\hline Nanjing & 0.757 & 0.897 & 0.661 & 0.749 & 0.961 \\
Suzhou & 0.643 & 0.840 & 0.914 & 0.693 & 0.956 \\
Wuxi & 0.618 & 0.777 & 0.815 & 0.639 & 0.864 \\
Changzhou & 0.504 & 0.655 & 0.690 & 0.614 & 0.660 \\
Zhenjiang & 0.453 & 0.584 & 0.613 & 0.588 & 0.597 \\
Nantong & 0.458 & 0.512 & 0.477 & 0.500 & 0.483 \\
Taizhou & 0.440 & 0.505 & 0.591 & 0.546 & 0.754 \\
Yangzhou & 0.244 & 0.431 & 0.441 & 0.489 & 0.582 \\
Xuzhou & 0.323 & 0.455 & 0.512 & 0.600 & 0.618 \\
Huaian & 0.241 & 0.255 & 0.353 & 0.388 & 0.424 \\
Yancheng & 0.194 & 0.245 & 0.311 & 0.385 & 0.262 \\
Suqian & 0.143 & 0.204 & 0.240 & 0.268 & 0.392 \\
Lianyungang & 0.164 & 0.200 & 0.185 & 0.198 & 0.233 \\
\hline
\end{tabular}

Table 5

Dynamic synergy degree of composite system in cities of Jiangsu obtained using bootstrap DEA.

\begin{tabular}{lccccc}
\hline \multirow{2}{*}{ Region } & \multicolumn{5}{c}{ Year } \\
\cline { 2 - 6 } & 2014 & 2015 & 2016 & 2017 & 2018 \\
\hline Nanjing & 0.757 & 0.827 & 0.772 & 0.766 & 0.805 \\
Suzhou & 0.643 & 0.741 & 0.799 & 0.772 & 0.809 \\
Wuxi & 0.618 & 0.698 & 0.737 & 0.713 & 0.743 \\
Changzhou & 0.504 & 0.580 & 0.617 & 0.616 & 0.625 \\
Zhenjiang & 0.453 & 0.518 & 0.550 & 0.559 & 0.567 \\
Nantong & 0.458 & 0.485 & 0.482 & 0.487 & 0.486 \\
Taizhou & 0.440 & 0.473 & 0.512 & 0.521 & 0.567 \\
Yangzhou & 0.244 & 0.338 & 0.372 & 0.401 & 0.437 \\
Xuzhou & 0.323 & 0.389 & 0.430 & 0.472 & 0.502 \\
Huaian & 0.241 & 0.248 & 0.283 & 0.309 & 0.332 \\
Yancheng & 0.194 & 0.220 & 0.250 & 0.284 & 0.280 \\
Suqian & 0.143 & 0.174 & 0.196 & 0.214 & 0.249 \\
Lianyungang & 0.164 & 0.182 & 0.183 & 0.186 & 0.196 \\
\hline
\end{tabular}

and Yancheng is between 0.3 and 0.4, indicating that the three cities are basically in a noncooperative state synergy. This level of synergetic development is lower than that for the other cities. The average state synergy degree of the other six cities is between 0.5 and 0.7 , indicating that the composite system is in a basic state synergy. The synergy of the composite system changes from year to year and the regional differences have increased over time. This reflects the fact that the composite system is affected by factors of social and economic development of both subsystems. Except for Nanjing, Suzhou, and Wuxi, the state synergy of the cities is below 0.8. This indicates that the level of synergetic development of the industrial agglomeration and urbanization composite system in Jiangsu is generally not high. 


\section{Conclusion and Suggestions}

After the data collection and processing of various indicators of the sensing system, we used a multivariate data analysis measurement model for comparison between regional industrial agglomeration and urbanization. We used bootstrap DEA and fuzzy mathematics to build a synergy effect model of an industrial agglomeration and urbanization composite system based on a self-organization theory using Jiangsu, China, as an example of an empirical study. The order parameters were used to analyze the level of synergetic development of industrial agglomeration and urbanization. From the analysis, we obtained the following conclusions.

(1) With the constant improvement of the level of social and economic development, the industrial agglomeration of Jiangsu is showing a maturing trend. The level of urbanization development has been fluctuating but the trend is upward.

(2) The levels of synergetic development of the industrial agglomeration and urbanization composite system and the two subsystems are also affected by socioeconomic factors and show regional differences.

(3) The level of synergetic development of the industrial agglomeration and urbanization composite system in Jiangsu is not high and has not reached a stable state. Increasing the degree of synergy has greatly promoted the development of the regional social economy.

On the basis of the above analysis, the following suggestions are given to further enhance the level of synergetic development of industrial agglomeration and urbanization, promoting the synergetic and sustainable development of the regional social economy.

(1) Led by the government, the forward-looking planning and positioning of industries and cities are required. Incorporating industrial agglomeration development into the overall urban planning should become the focus of urbanization, so that industrial agglomeration and urbanization result in organic and holistic synergetic development.

(2) The industrial agglomeration and synergetic development of urbanization should be enhanced. The implementation of an industrial agglomeration strategy will positively cultivate leading industrial clusters of cities and towns, promoting urban economic development. The evolution of industrial agglomeration is needed to improve the quality of urban life through an agglomeration strategy. This will also optimize the provision of industrial facilities, actively cultivating characteristic industrial cluster towns.

(3) The process of regional economic integration needs to be accelerated to promote industrial agglomeration and city development between economically well-developed and lessdeveloped regions. The developed regions are expected to transfer land-resource-intensive and labor-intensive industries to less-developed peripheral regions over time to promote high-tech industries and the strategic emergence of information resource gathering, information network construction, and new markets. This will lead to the sustainable development of industrial agglomerations and the increased competitiveness of cities. Future research will follow the development of new types of sensors. The application of big data for intelligent monitoring systems can provide city managers with more effective urban governance. 


\section{References}

1 T. Kabiraj and C. C. Lee: Int. Econ. J. 18 (2004). https://doi.org/10.1080/1016873042000270018

2 L. Simar and P. W. Wilson: Inst. Oper. Res. Manage. Sci. 44 (1998) 49. https://pubsonline.informs.org/doi/ abs/10.1287/mnsc. 44.1 .49

3 G.-y. Lu, X.-y. Fu, and S.-e. Zhu: Sch. Econ. Manage. 12 (2011) 101. https://kns.cnki.net/kcms/detail/detail. aspx?dbcode $=$ CJFD\&dbname $=$ CJFD2011\&filename $=$ ZGRK201112011\&v $=y z$ zxjajLQtmncGRC $\% 25 \mathrm{mmd} 2 \mathrm{FS}$ cjY7HAPUS\%25mmd2F9donHtJs50XS6c6m8NecbgZzSsxAap3D3y4B

4 W. Zhang, X. Huang, Y. Zheng, and X. Wang: Res. Econ. Manage. 37 (2016) 28. https://ie.cnki.net/kcms/ detail/detail.aspx?filename $=J J Y G 201602005 \&$ dbcode $=$ NCGJ\&dbname $=$ NCGLLKCJFDLAST2016\&v $=$

5 P. Jing: Urban Dev. Res. 18 (2011) 15. https://kns.cnki.net/kcms/detail/detail.aspx?dbcode=CJFD\&dbname=CJ FD2011\&filename $=$ CSFY201111007\&v $=$ g5RtLdbMkGCUTTuyZbRrnHRNcPhydQinSNoKDP\%25mmd2BU2 qW\%25mmd2FJbGTk\%25mmd2BVxG\%25mmd2FRTPHRoG\%25mmd2Fc2

6 I. Olkin and A. R. Sampson, Multivariate Analysis: Overview (Department of Statistics Stanford University, California, USA, 2001). https://statistics.stanford.edu/sites/g/files/sbiybj6031/f/2001-02.pdf

7 A. Marshall: Principles of Economics: Unabridged Eighth Edition (Cosimo, Inc., New York, 2009).

8 K. J. Button: Urban Economics: Theory and Policy (The MacMillan Press, London, 1976).

9 E. S. Mills and B. W. Hamilton: Urban Economics (HarperCollins Publishers, New York, 1994).

10 T. Dong, G. Zheng, B. Shen, and H. Wang: Faculty of Landscape Garden and Archit. 9 (2015) 30. https://kns. cnki.net $/ \mathrm{kcms} /$ detail $/$ detail.aspx?dbcode $=$ CJFD $\&$ dbname $=$ CJFDLAST2015\&filename $=$ YNNS201504006\&v $=$ dAntjW\%25mmd2BbSU7TjkuKg\%25mmd2BV427y7U1Z2CRyg\%25mmd2BGT7aCNSo1clc3sjpAw78MvCv rsoNtgu

11 S. Zhang, Y. Wang, and T. Chang: Sens. Mater. 30 (2018) 565. https://doi.org/10.18494/SAM.2018.1770

12 X.-R. Zhang and Z.-B. Wang: China's Popul. Resour. Environ. 22 (2012) 130.

13 H. Ding and H. Zheng: Coll. Econ. Meanage. 30 (2016) 79. https://kns.cnki.net/kcms/detail/detail.aspx?dbcode $=$ CJFD\&dbname $=$ CJFDLAST2016\&filename $=$ HDJJ201608013\&v=wE1iBfgOwbUW8i5grcJNFKd4rIpHZeSe fQb1TLmbNZv4luOS0801nPTI3\%25mmd2BNntrd\%25mmd2B

14 S. Duan, L. Zhang, and W.-L. Hsu: Sens. Mater. 32 (2020) 1913. https://doi.org/10.18494/SAM.2020.2719

15 R. Li, L. Qiang, and J.-m. Cai: Stat. Inf. Forum 29 (2014) 70. https://kns.cnki.net/kcms/detail/detail. aspx?dbcode $=$ CJFD \&dbname $=$ CJFD2014\&filename $=$ TJLT201403014\&v $=$ bparbwjMr72gVJuh5OzRLmfKQ7 6A1cLPUpyLzImO5IaUuuISPyph3GpPjCXrVRD\%25mmd2F

16 C. C. Fan and A. J. Scott: Econ. Geogr. 79 (2003). https://onlinelibrary.wiley.com/doi/abs/10.1111/j.19448287.2003.tb00213.x

17 F.-d. Qiu, C.-g. Zhu, and Z. Liu: Hum. Geogr. 6 (2006) 119. https://kns.cnki.net/kcms/detail/detail.aspx?dbcode $=$ CJFD $\&$ dbname $=$ CJFD2006\&filename $=$ RWDL200606026\&v $=$ B4E9ZpB6Q0pPoGIHO3f $\% 25 \mathrm{mmd} 2 \mathrm{BDczlC}$ 3cEcYddrYqYJGBfxqC3Wd3R43oKgcQBcOHHfB6s

18 A.-p. Hu, S.-f. Qiu, and D.-z. Wu: Logistical Eng. Univ. PLA. 30 (2016) 140. https://kns.cnki.net/kcms/detail/ detail.aspx ?dbcode $=$ CJFD $\&$ dbname $=$ CJFDLAST2016\&filename $=$ CGGL201603024\&v $=h$ hNWYGvVHtFQEx aqYpt14OLy73PFcCjtYmwREy21wEfHN9Ny1G4SXmBHE9wXaIao

19 P. Wang and Q. Wang: J. Hunan Agric. Univ. (Social Sci.) 17 (2016) 73. https://kns.cnki.net/kcms/detail/detail. aspx?dbcode $=$ CJFD $\&$ dbname $=$ CJFDLAST2016\&filename $=$ HNNS201602012 \&v $=J$ GEg $4 \mathrm{hhOUK} \% 25 \mathrm{mmd} 2 \mathrm{~B}$ Cdj054nlyTe5dsTLqK\%25mmd2BNDB6RpUObsrglukMKTADg7mM\%25mmd2FnXM\%25mmd2FhEhuZ 